\title{
UPPER BOUND ON OUTAGE CAPACITY OF ORTHOGONAL RELAY NETWORKS
}

\author{
Jesus Gómez-Vilardebó ${ }^{1}$ and Ana I. Perez-Neira ${ }^{2}$ \\ ${ }^{1}$ Centre Tecnolòic de Telecomunicacions de Catalunya (CTTC) \\ Parc Mediterrani de la Tecnologia, Av. Canal Olímpic s/n, 08860 Castelldefels (Barcelona, Spain) \\ jesus.gomez@cttc.es \\ ${ }^{2}$ Signal Processing and Communications Department \\ Technical University of Catalonia (UPC) (Barcelona, Spain) \\ anuska@gps.tsc.upc.edu
}

\begin{abstract}
We consider the orthogonal multiple relay channel at the low $S N R$ regime.The source transmits to the destination and all the possible relays in one channel and the relays transmit in different and orthogonal channels making use of the transmission received from the source. This cooperative strategy achieves diversity gains at a high cost in bandwidth efficiency, due to the orthogonality of the transmissions. However, it has the desired benefit of allowing all the nodes in the network to behave as in a point to point channel. Based on the max-flow min-cut upper bound for relay networks, we explore the capacity limits of this kind of communication, obtaining an upper bound on the capacity when full channel state information (CSI) is available at every network node. This focus gives insight on the design of the transmitting strategy and define how feedback from relays and destination can be performed practically and optimally, giving ideas for higher layer protocols.
\end{abstract}

\section{INTRODUCTION}

Wireless medium is intrinsically broadcast, as information flows towards all the terminals at a reachable distance. An inherent cooperative feature can be exploited without any additional infrastructure, as opposed to the wired medium. The pioneer work of [1] formulate that information flow as the so-called relay channel, where some intermediate nodes help in forwarding the message; thus, originating the so needed diversity in fading channels. Within cooperative networks, space diversity is created using a collection of distributed antennas belonging to multiple users, each with their own information to transit. In the literature, this form of space diversity is referred to as cooperative diversity, because the terminals share their antennas and other resources to create "virtual array" through distributed transmission and signal processing. There has been much interest in channels with orthogonal transmissions. In many practical wireless systems, nodes cannot transmit and receive at the same time and over the same frequency, that's called half duplex constraint. Furthermore, in cooperative systems, assuming perfect synchronization of independent transmitters is a

This work was partially supported by the Catalan Government under grant SGR2005-00996; by the Spanish Government under projects TEC2005-08122-C03 and FIT 330220-2005-111 (PlaNetS); and by the European Commission under project IST-2002-2.3.1.4 (NEWCOM).

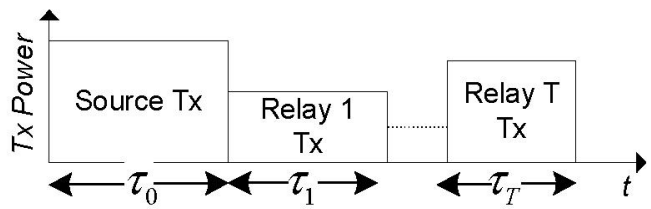

Fig. 1. Example of the time-division channel allocation for the orthogonal cooperative transmissions considered in this paper.

very hard requirement, even in that case, allowing to receive simultaneous signals from different nodes, comes at a high cost in receiver complexity, because symbol synchronization and channel estimation must be performed, jointly, to all the incoming paths as in a MSO channel [2].

Analysis of cooperative strategies usually needs to assume an specific transmission scheme at relays; Amplify and Forward and Decode and Forward are the more typical ones. This is the focus in [3] for one relay and in [4] for network relaying using distributedspace time codes. In this works, orthogonal relaying strategies were considered at high $S N R$ and no CSI was available at the transmitters side. In [5] those strategies were extended to only half-duplex constraint, which turns to be much less restrictive and offer better performance. We will not assume any specific transmission scheme apart from forcing all transmissions to occur in orthogonal channels, as in the time-division model despicted in Fig 1. Our starting point is the so called max-flow min-cut upper bound for relay networks [6, p. 445]. Despite of being an upper bound, it has been proved to be achievable in many specific cases. The most well known, is the so called degraded relay channel [1] in which the relay is forced to decode the source transmitted message. However Decode and Forward relaying turns to be a very hard constraint at the low $S N R$ regime. In fact, in the case of low $S N R$, [7] proves that a very simple transmission strategy, where the source transmits with a low duty cycle but at high power, while the relay transmits with the simple Amplify and Forward scheme, achieves the upper bound. As stated in [7], diversity gain is more significant at low than at high $S N R$, as it also occurs with CSI at transmitters side. Although, assuming perfect CSI at every network node could be a quite hard requirement for our desired low 


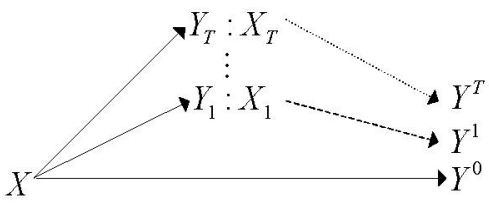

Fig. 2. Communication model used in this paper. In the case of orthogonal transmissions, channels from source or any of the relays works in different frequency, time or orthogonal code.

complexity scheme, it allows us to define how feedback from relays and destination can be performed optimally, giving ideas for higher layer protocols. Results show that, in the orthogonal relaying case considered, a very simple threshold criterion is enough to select the optimal set of relaying nodes. Furthermore, full CSI at every network node is not necessary to perform optimal energy allocation. The source needs few feedback from the selected relays and relays just only need their own local CSI.

The remainder of the paper is organized as follows: In section 2 the Orthogonal Relay Network Model at low $S N R$ is described in terms of mutual information and the max-flow min-cut upper bound is particularized for wireless networks under consideration. In section 3 the problem is formulated and solved as a linear problem with linear constraints and then the main results are stated, within a comprehensive explanation of its behavior. Finally in section 4 some simulation results are shown.

\section{SYSTEM MODEL}

In this section we will first present the cut set upper bound for discrete memoryless relay networks, which will be the starting point of our analysis. Then the bound will be characterized for the special case of the orthogonal relay network model considered in this paper. Finally the low $S N R$ approximation will be derived

\subsection{General Relay network Model}

We consider the transmission of a message $X$ from a source terminal to a destination terminal which receives $Y$, using up to $T$ relaying nodes, see Fig. 2. Denote $\mathcal{T}=\{1,2, \ldots, T\}$ as the set containing all possible relays, $\mathcal{S}$ as any of the $2^{T}$ possible subsets that can be construct with up to $T$ relays and $\mathcal{S}^{C}$ the complement of $\mathcal{S}$ in $\mathcal{T}$. For example, in the case of the two relays network, there exist 4 possible cuts: $\mathcal{S}=\{0\}, \mathcal{S}=\{1,2\}, \mathcal{S}=\{2\}$ and the one depicted in Fig. $3, S=\{1\}$. Denote $X_{\mathcal{S}}=\left\{X_{t}: t \in \mathcal{S}\right\}$ and $Y_{\mathcal{S}}=\left\{Y_{t}: t \in \mathcal{S}\right\}$ as the transmitted and received signals at the relaying nodes for a given subset $S$. A capacity upper bound for this relaying network model is given by the cut-set bound in [8]

$$
C \leq \max _{p\left(x, x_{1}, \ldots, x_{T}\right)} \min _{S \subseteq T} I\left(X, X_{\mathcal{S}} ; Y_{\mathcal{S}^{C}}, Y \mid X_{\mathcal{S}^{C}}\right)
$$

where $p\left(x, x_{1}, \ldots, x_{T}\right)$ is the channel inputs distribution and $I(X ; Y)$ is the mutual information between $X$ and $Y$. Note that (1) define a convex optimization problem, the set of $p\left(x, x_{1}, \ldots, x_{T}\right)$ is convex, and the mutual information expressions are concave in $p\left(x, x_{1}, \ldots, x_{T}\right)$. Furthermore, the point-wise

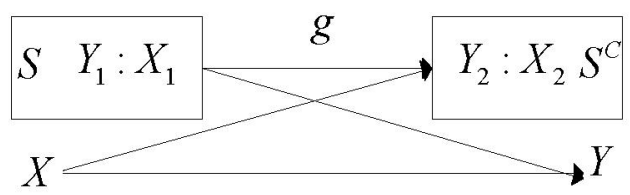

Fig. 3. Possible cut in the two-relay general network. $\mathcal{S}=\{1\}$, $\mathcal{S}^{C}=\{2\}$. Transmitting nodes: Source and relay 1. Receiving nodes: Relay 2 and Destination.

minimum of a collection of concave functions is concave. Equation (1) states that the capacity of a relaying network can not be greater than the capacity of any of the virtual MIMO channels that can be created employing the relays antennas at the transmitter or receiver side, conditioned to the information being sent by the receiving relays.

\subsection{Orthogonal Relay Network Model}

Consider now the special case where all the transmissions from source or relays to destination occur in orthogonal channels, as the one depicted in Fig. 1. Denote $\tau_{i}$, as the fraction from the total (frequency or time) channel allocated to the transmission of node $i$. Without loss of generality, in this paper, we focus on a time division model for which it is not needed any additional hardware as opposed to the frequency division model. For this orthogonal relay network, the mutual information associated to a given cutset $\mathcal{S}$ can be rewritten as

$$
\begin{aligned}
& I\left(X, X_{\mathcal{S}} ; Y_{\mathcal{S}^{C}}, Y \mid X_{\mathcal{S}^{C}}\right) \\
& \quad=I\left(X, X_{\mathcal{S}} ; Y_{\mathcal{S}^{C}}, Y\right) \\
& =\tau_{0} I\left(X ; Y_{\mathcal{S}^{C}}^{0}, Y^{0}\right)+\sum_{i \in \mathcal{S}} \boldsymbol{\tau}_{i} I\left(X_{i} ; Y_{\mathcal{S}^{C}}^{i}, Y^{i}\right) \\
& =\tau_{0} I\left(X ; Y_{\mathcal{S}^{C}}^{0}, Y^{0}\right)+\sum_{i \in \mathcal{S}} \boldsymbol{\tau}_{i} I\left(X_{i} ; Y^{i}\right) .
\end{aligned}
$$

The proof follows similar argument to [9, Appendix A]. The proof interpretation could be as follows, by the orthogonality of the transmissions, channel inputs $X_{\mathcal{S}}$ take place in different channels to $X_{\mathcal{S}}$ ones and mutual information in (2) can be rewritten as in (3). Furthermore (3) can be decomposed in a sum of $|\mathcal{S}|+1$ broadcast SIMO channels with one channel input and $\left|\mathcal{S}^{C}\right|$ channel outputs (4), where $|\cdot|$ denotes cardinality. Here we have made use of notation $Y_{S C}^{i}$ to emphasize that the output signal at the relay nodes $\mathcal{S}^{C}$ from the transmitting node $i$, takes place in channel use $i$. Finally, when constraining the relays terminals only to make use of source transmitted signal but not the one from the other relays (i.e., channel $g$ in Fig. 3 is not considered) mutual information simplifies to (5). In that case, the maximum average mutual information in the bound is obtained by each node employing independent random codebooks generated independent and identically distributed (i.i.d.) circularly symmetric, complex Gaussian. Thus the $c u t$-set capacity, associated to the set $\mathcal{S}$, is 


$$
\begin{aligned}
C_{S} & =\boldsymbol{\tau}_{0} \ln \left(1+P_{0} \alpha_{0}+P_{0} \sum_{i \in \mathcal{S}^{C}} \alpha_{i}\right) \\
& +\sum_{i \in \mathcal{S}} \boldsymbol{\tau}_{i} \ln \left(1+P_{i} \beta_{i}\right) .
\end{aligned}
$$

The network we consider is memoryless and time invariant during the transmission of a codeword. For convenience we have denote $\alpha_{i}$ as the channel gain from the source terminal to the $i t h$ relay terminal, $\beta_{i}$ as the channel gain from the relay terminal $i t h$ to the destination, $\alpha_{0}$ as the gain of the direct channel between source and destination, and $P_{i}$ as the mean transmitted power allocated to the transmission of node $i$. For simplicity, but without loss of generality, we consider a discrete-time channel to avoid introducing the notation associated with time and bandwidth. In that way, we assume that all quantities $\alpha_{0}, \alpha$ and $\beta$ are normalized so that the sampled receiver noise has unit variance. In this paper we compute the mutual information and rate in nats/s.

\subsection{The low SNR regime}

As stated before, we will consider the low $S N R$ regime, in that regime (6) can be approximated as

$$
C_{S} \simeq E_{0} \alpha_{0}+E_{0} \sum_{i \in \mathcal{S}^{C}} \alpha_{i}+\sum_{i \in \mathcal{S}} E_{i} \beta_{i} .
$$

where we have denoted $E_{i}=\tau_{i} P_{i}$ as the energy allocated to transmitting node $i$. Denoting the vector of allocated energies as $\mathbf{E}$, the problem defined in (1) can be rewritten as

$$
C \leq \max _{\mathbf{E}} \min _{S \subseteq T} C_{S} .
$$

Note that, in the low SNR regime, energy can be controlled either by the time interval or by the transmitted power, i.e. we can force all the transmission intervals to be equal to $\tau_{0}=\frac{1}{|\mathcal{S}|+1}$ and changing the mean transmitted power $P_{i}$. Furthermore, note that, those expressions were obtained from independent codebooks at the relays. If we consider repetition coding, where each relay employs exactly the same codebook as the source, and the virtual receiver perform optimal (maximal-ratio) combing, the instantaneous capacity associated to a cut-set $\mathcal{S}$, is given by $C_{\mathcal{S} \text {, rep }}$

$$
C_{S, \text { rep }}=\tau_{0} \ln \left(1+P_{0} \alpha_{0}+P_{0} \sum_{i \in \mathcal{S}^{C}} \alpha_{i}+\sum_{i \in \mathcal{S}} P_{i} \beta_{i}\right) .
$$

for which low SNR approximation is equal to the one obtained assuming independent codebooks at the relays in (7). Thus, at low $S N R$ repetition coding is optimal.

\section{OPTIMAL RESOURCE ALLOCATION IN THE LOW SNR REGIME}

Throughout the paper, we use optimal resource allocation to refer to the vector $\mathbf{E}$ and the subset $\mathcal{S}$, that maximize the upper bound in (8). We start by finding the set of conditions that ensure that the cut $\mathcal{S}$ is the set that obtain the minimum capacity among all possible cuts allowing us not to consider the internal minimization.

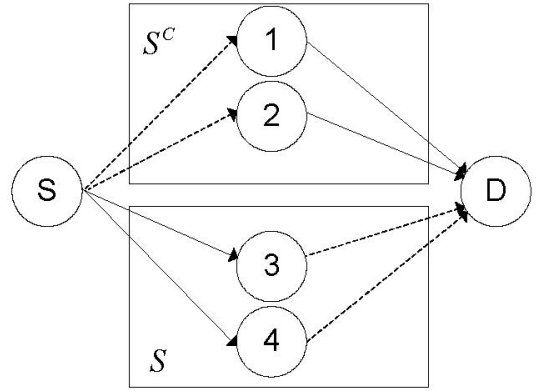

Fig. 4. Graphical illustration of the conditions that must hold whenever the capacity cut $\mathcal{C}_{\mathcal{S}}$ turns to be the minimum one. Weak channel are indicated by dashed lines and the strong channels with solid lines.

Theorem 1 The cut-set $\mathcal{S}$, in the case of orthogonal transmissions and low $S N R$, is the one that minimize the bound among all the possible cuts, if and only if

$$
\begin{array}{ll}
E_{0} \alpha_{i}-E_{i} \beta_{i} \leq 0 & \forall i \in \mathcal{S}^{C} \\
E_{i} \beta_{i}-E_{0} \alpha_{i} \leq 0 & \forall i \in \mathcal{S}
\end{array}
$$

Proof. Denote $\mathcal{S}$ as the minimum cut and $\mathcal{R}$ as any other possible cut different to $\mathcal{S}$. We want to obtain necessary and sufficient conditions that ensure $C_{S} \leq C_{R}$, in that case, using (7), for any subset $\mathcal{R}$, the following condition must hold

$$
E_{0}\left(\sum_{i \in \mathcal{S}^{C}} \alpha_{i}-\sum_{i \in \mathcal{R}^{C}} \alpha_{i}\right) \leq \sum_{i \in \mathcal{R}} E_{i} \beta_{i}-\sum_{i \in \mathcal{S}} E_{i} \beta_{i},
$$

removing all the common terms in $\mathcal{S}$ and $\mathcal{R}$ and also those in $\mathcal{S}^{C}$ and $\mathcal{R}^{C}$, we obtain

$$
E_{0}\left(\sum_{i \in \Omega_{1}} \boldsymbol{\alpha}_{i}-\sum_{i \in \Omega_{2}} \boldsymbol{\alpha}_{i}\right) \leq \sum_{i \in \Omega_{1}} E_{i} \beta_{i}-\sum_{i \in \Omega_{2}} E_{i} \beta_{i},
$$

where, $\Omega_{1}=\mathcal{S}^{C} \cap \mathcal{R}$ and $\Omega_{2}=\mathcal{R}^{C} \cap \mathcal{S}$.

The first step is to look for a set of necessary conditions. For each $i \in \mathcal{S}^{C}$, we could choose the set $\mathcal{R} \triangleq \mathcal{S} \cup\{i\}$. In that case: $\Omega_{1}=\mathcal{S}^{C} \cap \mathcal{R}=\{i\}$ and $\Omega_{2}=\mathcal{R}^{C} \cap \mathcal{S}=\varnothing$, forcing to hold conditions in (10). Reversely, for each $i \in \mathcal{S}$ we could choose the set $\mathcal{R}^{C} \triangleq \mathcal{S}^{C} \cup\{i\}$. In that case: $\Omega_{1}=\mathcal{S}^{C} \cap \mathcal{R}=\varnothing$ and $\Omega_{2}=\mathcal{R}^{C} \cap \mathcal{S}=\{i\}$, forcing conditions in (11) to hold. Finally, it remains to show that (10) and (11) are also sufficient conditions. Because they must hold and $\Omega_{1} \subset S^{C}$ and $\Omega_{2} \subset S$, inequation (13) also holds and so does (12).

Remark 1 Theorem 1 has a very intuitive interpretation. A subset $\mathcal{S}$ will produce the minimum capacity whenever all the relay nodes included in $\mathcal{S}$ have a better channel from the source than the one they have to the destination and reversely all the relays belonging to $\mathcal{S}^{C}$ have poor channel from the source than the one they have to the destination. A graphical interpretation of this behavior is represented in Fig. 4. 
Theorem 1 allows us to solve optimization problem (8) in two steps. As the internal minimization can be removed by imposing constraints (12) and (13) for a given cut set $\mathcal{S}$, the external maximization in $\mathbf{E}$ can be solved as a linear problem with linear constraints. Once this maximization is performed, it only remains to choose the subset $\mathcal{S}$ that maximize the upper bound on the capacity. Thus, taking into account that the capacity $C_{S}$ is a linear function of the energy from the source and all the relays belonging to $\mathcal{S}$. The maximization of $C_{S}$ can be written as the following convex minimization problem, in which the maximization in (8) within the convex (linear) region defined by $(10),(11)$ and the total energy constraint, has been written as the minimization of the same function changing its sign. The problem can be written in convex form as

$$
\begin{aligned}
C<-i \operatorname{Sn}_{\mathbf{S}} \min _{\mathbf{E}}-E_{0} \alpha_{0}-E_{0} \sum_{i \in \mathcal{S}^{C}} \alpha_{i}-\sum_{i \in \mathcal{S}} E_{i} \beta_{i} \\
\text { s.t. } \quad E_{0} \alpha_{i}-E_{i} \beta_{i} \leq 0 \quad \forall i \in \mathcal{S}^{C} \\
E_{i} \beta_{i}-E_{0} \alpha_{i} \leq 0 \quad \forall i \in \mathcal{S} \\
\sum_{i \in\{0, T\}} E_{i}-E=0
\end{aligned}
$$

where we have force a total transmitted energy constraint $E$, which means that we are looking for the optimal resource allocation in terms of minimal total consumed energy. Solution of this problem is stated in the following theorem.

Theorem 2 An upper bound for the capacity of orthogonal relay network with channel state information and total transmitted energy constraint, is given by

$$
C \leq E \frac{\alpha_{0}+\sum_{i \in \mathcal{S}^{* C}} \alpha_{i}}{1+\sum_{i \in \mathcal{S}^{*} C} \frac{\alpha_{i}}{\beta_{i}}}=E \eta^{*} .
$$

where $\mathcal{S}^{*}$ is the subset of $\mathcal{T}$, that satisfies $\mathcal{S}^{*}=\left\{i: \eta^{*} \geqslant \beta_{i}\right\}$.

Proof. The proof is done in two steps, firstly from the KKT condition the internal minimization in (14) is solved to any subset $\mathcal{S}$, then necessary and sufficient conditions for any node to belong to the optimal cut $\mathcal{S}^{*}$ are obtained. Denoted $\mathcal{R}$ as the subset of $\mathcal{S}$ that contains the nodes that satisfy $\eta_{\mathcal{S}}^{*} \leq \beta_{i}$ and $\mathcal{N}$ as the subset of $\mathcal{S}$ that contains the nodes that do not satisfy $\eta_{\mathcal{S}}^{*} \leq \beta_{i}$. Once we have solved the KKT conditions, the capacity of a given set $\mathcal{S}$, is

$$
C_{\mathcal{S}} \leq E \frac{\alpha_{0}+\sum_{i \in \mathcal{S}^{C}} \alpha_{i}+\sum_{i \in \mathcal{R}} \alpha_{i}}{1+\sum_{i \in \mathcal{S}^{C}} \frac{\alpha_{i}}{\beta_{i}}+\sum_{i \in \mathcal{R}} \frac{\alpha_{i}}{\beta_{i}}}=E \eta_{\mathcal{S}}^{*} .
$$

and the power allocation is given by

$$
\begin{aligned}
& E_{0}^{*}=\frac{E}{1+\sum_{i \in \mathcal{S} C} \frac{\alpha_{i}}{\beta_{i}}+\sum_{i \in \mathcal{R}} \frac{\alpha_{i}}{\beta_{i}}} \\
& E_{i}^{*}=\frac{\alpha_{i} \in \mathcal{S}^{C}}{\beta_{i}} E_{0}^{*} \quad \forall i \in\left\{\mathcal{R}, \mathcal{S}^{C}\right\} \\
& E_{i}^{*}=0 \quad \forall i \in \mathcal{N}
\end{aligned}
$$

It remains to find the optimal cut $\mathcal{S}$ that maximize $\eta_{\mathcal{S}}^{*}$. For this purpose, it follows from (16), that if there exist some node indexed by

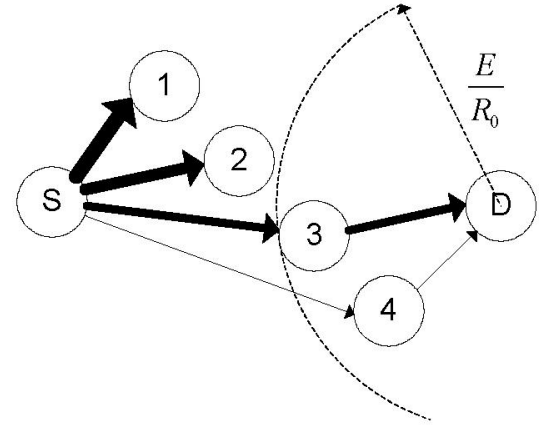

Fig. 5. Graphical interpretation for the selection of the transmitter set and the energy allocated at the relays.

$x$ inside $\mathcal{S}^{C}$, that do not satisfy $\eta_{\mathcal{S}}^{*} \leq \beta_{x}$, the capacity $C_{\mathcal{S} \cup\{x\}}$ will be greater than $C_{\mathcal{S}}$ if $\eta_{\mathcal{S} \cup\{x\}}^{*} \leq \beta_{x}$. Thus all nodes inside $\mathcal{S}^{*}$ must satisfy $\eta^{*} \geqslant \beta_{i}$ and all nodes inside $\mathcal{S}^{C *}$ must satisfy $\eta^{*} \leq \beta_{i}$.

Remark 2 The energy allocation that achieves the capacity (15) is

$$
\begin{array}{ll}
E_{0}^{*}=\frac{E}{1+\sum_{i \in \mathcal{S}^{* C}} \frac{\alpha_{i}}{\beta_{i}}} & \\
E_{i}^{*}=\frac{\alpha_{i}}{\beta_{i}} E_{0}^{*} & \forall i \in \mathcal{S}^{C} \\
E_{i}^{*}=0 & \forall i \in \mathcal{S}
\end{array}
$$

Note that relaying energy transmission depends only on local information at the relaying nodes. That is, on their instantaneous received energy from the source $\alpha_{i} E_{0}^{*}$. Furthermore source terminal can determine the optimum energy to transmit from $\frac{\alpha_{i}}{\beta_{i}}$ feedback of the relays nodes which will participate in the communication.

The above solution can be implemented in systems with a desired fixed transmission rate $R_{0}$. The energy allocation in (17) determines that only relays inside $\mathcal{S}^{*, C}$, will participate in the communication. Those relays must satisfy the condition $\eta^{*} \leq \beta_{i}$, which in case of fixing the desired rate to be $R_{0}$ is equal to satisfying $\frac{R_{0}}{E} \leq \beta_{i}$. Note that this selection of the transmitter set only depends on the channel between the relaying nodes and the destination. This is the opposite behavior of Decode and Forward strategies, in which only relay nodes that have been successful in decoding the message will participate in the communication, it means $\alpha_{i}$ must be greater than some design threshold parameter. Fig. 5 explains the practical behavior of this strategy. Only for graphical interpretation, we understand that more proximity means better channel and that line thickness represents the amount of received energy. Condition $\frac{E}{R_{0}}>\beta_{i}$ establishes a region that determines the nodes which will participate in the communication. Note that relays 1,2 in Fig. 5 have better channel from the source, but they do not transmit any signal because of the energy cost of the transmission to destination. Relaying nodes must allocate as much energy as needed to ensure that destination receive the same energy as the one they have received from the source. Intuitively, it states that if a relaying node receive very low $S N R$ it must not amplify very much the signal because it carries a lot of noise. The converse occurs if it receives at high $S N R$. 


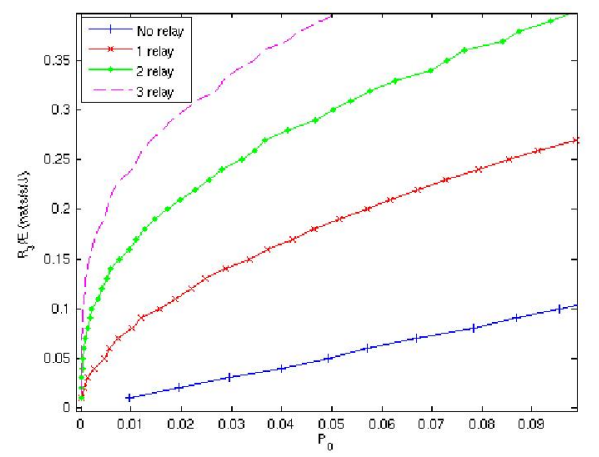

Fig. 6. Outage Capacity per unit energy $\eta^{*}=\frac{R_{0}}{E}$ as a function of the desired outage probability $P_{0}$ and different number of available relays.

\section{SIMULATION RESULTS}

In the simulations, it is considered the slow fading situation, where the delay requirement is short compared to the coherence time of the channel. The channel gains follow an exponential distribution, but they remain constant until all the relays have already ended its transmission. We focus the analysis on the Outage Capacity computed from a desired rate $R_{0}$, as $P_{0}=p\left(E \eta^{*}<R_{0}\right)$. Specifically we consider the Outage Capacity per Energy Unit, defined as $\frac{R_{0}}{E}=\eta^{*}$. Fig. 6, shows the Outage Capacity per Energy Unit as a function of the desired outage probability $P_{0}$, for a different number of available relays. While in the noncooperative transmission, $\eta^{*}$ grows linearly with $P_{0}$. The cooperative transmission appears to follow a $\sqrt[T+1]{P_{0}}$ rule. More interesting interpretation appears in Fig 7 where the outage probability has been fixed to $10^{-3}$. The minimum number of available relays and average number of active relays are plotted as a function of the Outage Rate per Energy Unit. As expected, it shows that whenever we want to increase the number of transmitted bits per unit energy, we need to increase the total number of available relays. What is more surprising is that despite of using better relays we still need to increase the number of retransmissions in average (active users) if we want to increase $\frac{R_{0}}{E}$. That means, that cooperation effectively improves the bandwidth inefficiencies of orthogonal retransmissions. Obviously, whenever there exists more available relays, the portion of those which are active is lower.

\section{CONCLUSIONS}

In this paper, we have studied the orthogonal multiple relay channel at the low $S N R$ regime, when full CSI is available at every network node. For this channel, a capacity upper bound, based on the max-flow min-cut upper bound for relaying networks is obtained. This approach gives us insight on the design of the transmitting strategy and define how feedback from relays and destination could be performed practically and optimally, giving ideas for higher layer protocols. Results show that a simple threshold criterion is enough to select the optimal set of relaying nodes. Furthermore, it is shown that full CSI at every network node is not

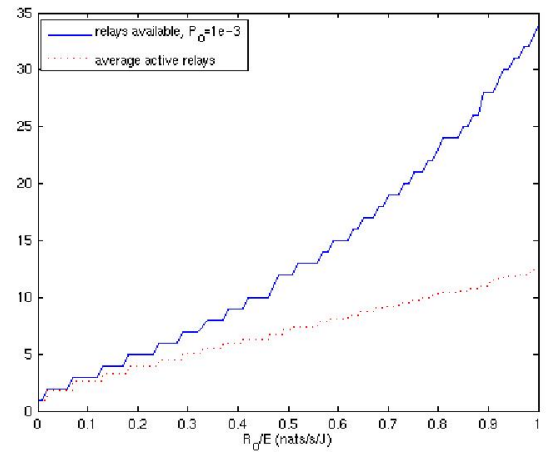

Fig. 7. Minimum number of available relays and average active relays that are needed to ensure $P_{0}$ less than $10^{-3}$.

necessary to perform optimal energy allocation. In fact, the source node needs few feedback from the selected relays and relays just only need its own local CSI

\section{REFERENCES}

[1] T. M. Cover and A. A. E. Gamal, "Capacity theorems for the relay channel," IEEE Trans. Inform. Theory, vol. 25, no. 5, pp. 572-584, Sept. 1979.

[2] A. Vosoughi and Scaglione, "On the effect of receiver estimation error upon channel mutual information," IEEE Trans. Inform. Theory, vol. 54, no. 2, pp. 459-472, Feb. 2006.

[3] J. N. Laneman, D. N. C. Tse, and G. W. Wornell, "Cooperative diversity in wireless networks: Efficient protocols and outage behavior," IEEE Trans. Inform. Theory, vol. 50, no. 12, Dec. 2004.

[4] J. N. Laneman and G. W. Wornell, "Distributed space-timecoded protocols for exploiting cooperative diversity in wireless networks," IEEE Trans. Inform. Theory, vol. 49, no. 10, pp. 2415-25.

[5] K. Azarian, H. E. Gamal, and P. Schniter, "On the achievable diversity-multiplexing tradeoff in half-duplex cooperative channels," IEEE Trans. Inform. Theory, vol. 51, no. 12, pp. 4152-4172, Dec.

[6] T. Cover and J. A. Thomas, Elements of Information Theory., New York: Wiley, 1991.

[7] A. S. Avestimehr and D. N. C. Tse, "Outage-optimal relaying in the low SNR regime," in Proc. IEEE Int. Symp. Information Theory (IST), Sept., pp. 941-945.

[8] A. A. E. Gamal, "On information flow in relay networks," in Proc. IEEE National Telecommunications Conf., vol. 2, Miami, FL, Nov. 1981, pp. D4.1.1-D4.1.4.

[9] A. Host-Madsen and J. Zhang, "Capacity bounds and power allocation for the wireless relay channel," IEEE Trans. Inform. Theory, vol. 51, no. 6, pp. 2020-2040, June 2005. 